Original Articles

\title{
Testing the causal theory of reference
}

\author{
Filippo Domaneschi ${ }^{\mathrm{a}, *}$, Massimiliano Vignolo ${ }^{\mathrm{b}}$, Simona Di Paola ${ }^{\mathrm{a}}$ \\ ${ }^{a}$ University of Genoa, DISFOR - Department of Educational Sciences, Psychology Unit, Corso Podestà, 2, Room 3C3, 16128 Genova, Italy \\ ${ }^{\mathrm{b}}$ Department of Philosophy, University of Genoa, Via Balbi 4, 16126 Genova, Italy
}

\section{A R T I C L E I N F O}

\section{Article history:}

Received 6 July 2016

Revised 23 December 2016

Accepted 28 December 2016

\section{Keywords:}

Experimental semantics

Theories of reference

Causal theory of reference

Semantic reference

Speaker's reference

\begin{abstract}
A B S T R A C T
Theories of reference are a crucial research topic in analytic philosophy. Since the publication of Kripke's Naming and Necessity, most philosophers have endorsed the causal/historical theory of reference. The goal of this paper is twofold: (i) to discuss a method for testing experimentally the causal theory of reference for proper names by investigating linguistic usage and (ii) to present the results from two experiments conducted with that method. Data collected in our experiments confirm the causal theory of reference for people proper names and for geographical proper names. A secondary but interesting result is that the semantic domain affects reference assignment: while with people proper names speakers tend to assign the semantic reference, with geographical proper names they are prompted to assign the speaker's reference.
\end{abstract}

(c) 2016 Elsevier B.V. All rights reserved.

\section{Introduction: philosophers' reactions to experimental semantics}

A theory of reference for proper names answers the following question:

(i) In virtue of what facts is an object $o$ the bearer of the name "N" as used by a speaker $\mathrm{S}$ ?

Since Saul Kripke published Naming and Necessity, most philosophers have endorsed the causal theory of reference. According to this theory, a speaker S refers to an object $o$ by using a name "N" when she is part of a communication chain that originated with a baptism or a grounding of the name " $\mathrm{N}$ " into the object $o$ and through which information about the object $o$ is transmitted. The crucial point in opposition to the classical descriptivist theory of reference is that the information that the speaker associates to the name might be incomplete, i.e., not detailed enough to single out the bearer of the name or even false of the bearer of the name. In particular, it is not the fact that the speaker associates a true identifying description of the bearer with the name that makes the name refer to the bearer.

The first works in experimental semantics addressed the methodological question concerning the evidence on which philosophers rely to establish which theory of reference is true.

\footnotetext{
* Corresponding author.

E-mail addresses: filippo.domaneschi@unige.it (F. Domaneschi), maxi@nous. unige.it (M. Vignolo), simona.dip@hotmail.com (S. Di Paola).
}

Machery et al. (2004, 2009) and Mallon, Machery, Nichols, and Stich (2009) have attacked the methodology of philosophers of language by assuming that it is ultimately grounded on the evidential role of intuitions. ${ }^{1}$ Philosophical theories of reference are correct to the extent that they are confirmed by competent speakers' referential intuitions. If a theory of reference says that in a given scenario, a proper name "N" refers to an object $o$, then the theory is confirmed if competent speakers have the intuition that in that scenario the proper name " $\mathrm{N}$ " refers to the object $o$. If competent speakers have the intuition that the proper name "N" does not refer to 0 , then the theory is disconfirmed.

In Machery et al.'s view, philosophers of language endorse the methodological principle called the method of cases, ${ }^{2}$ here reformulated as follows with regard to question (i):

The method of cases: the correct philosophical theory of reference for a proper name "N" is the theory that is best supported by the intuitions that competent speakers have about "N"'s reference.

This methodological principle presumes that semantic intuitions be uniformly shared among speakers and across actual and possible cases. Machery et al. (2004), Machery, Olivola, and de Blanc (2009) questioned the truth of this presumption. First, they noted that philosophers have exclusively relied on their own intuitions, i.e., theoreticians' intuitions. Second, by means of an empir-

\footnotetext{
1 Genone and Lombrozo (2012) presented an experimental survey based on the same assumption of Machery, Mallon, Nichols, and Stich (2004).

2 See Mallon et al. (2009).
} 
ical survey, they tried to show that semantic intuitions vary among speakers of different linguistic and cultural communities. In a series of experiments, they tested ordinary speakers' referential intuitions in cases modelled after Kripke's Gödel/Schmidt story. ${ }^{3}$ In Naming and Necessity, Kripke (1980) depicts a counterfactual scenario in which Gödel is not the author of the theorem of the incompleteness of arithmetic. Gödel stole the proof of the theorem from Schmidt, the author of the theorem in the counterfactual scenario. According to Kripke, in that scenario, a hypothetical speaker who associated the unique description "the author of the theorem of the incompleteness of arithmetic" with the name "Gödel" would still refer to Gödel and not to Schmidt. In Machery et al.'s interpretation, Kripke's refutation of classical descriptivism relies on the evidence that competent speakers share the intuition that in the counterfactual scenario the name "Gödel" refers to Gödel.

Machery et al. claim to have proven that there is no such evidence. They say that the empirical data they have collected show that competent speakers in different linguistic communities (e.g., a group of Western participants and a group of Eastern Chinese participants) do not share the intuition that the name "Gödel" refers to Gödel in the counterfactual scenario. They conclude that the philosophical methodology is severely flawed because it employs the method of cases, which in turn presupposes the uniformity of referential intuitions among speakers. However, speakers do not share the same referential intuitions. Consequently, philosophical theories of reference are baseless with regard to the kind of evidence that philosophers employ to support them.

Machery et al.'s experiments have generated two main strands ${ }^{4}$ of reactions from philosophers. Some commentators have strongly rejected Machery et al.'s view that referential intuitions play a crucial role in Kripke's refutation of classical descriptivism. According to Deutsch (2009, 2010, 2015), Ichikawa, Maitra, and Weatherson (2011) and Martí (2014), Machery et al.'s attack of the methodology in the philosophy of language originated from a mistaken interpretation of Kripke's Gödel/Schmidt story. These philosophers claim that the evidence in favour of Kripke's refutation of classical descriptivism derives, not from polling philosophers' or ordinary speakers' referential intuitions, but from the error and ignorance argument and the immunity from mistake argument. Hence, these philosophers argue that the target of Machery et al.'s attack is misplaced.

Other philosophers agree that the polemic target of Machery et al.'s empirical surveys is misplaced, but they are nonetheless concerned with the challenge raised by experimentalists. Such philosophers have taken seriously the idea that experiments may have an evidential role in the philosophy of language. Devitt (2011a, 2011b, 2015) and Martí (2009, 2014) have pointed out that the experiments conducted by Machery et al. do not elicit the kind of data that are relevant to the theory of reference. Devitt and Martí's objection is that Machery et al.'s tests invite participants to think about how a hypothetical speaker uses a name, and they prompt participants to reflect on how they think reference is determined. However, data on how participants think that reference is determined are not relevant for philosophers of language who try to explain it in virtue of what proper names refers to their bearers. Asking participants to reflect on whether the character of the vign-

\footnotetext{
${ }^{3}$ The classic version of descriptivism that Kripke refutes in Naming and Necessity is the view that names have their bearers semantically fixed by descriptions. The descriptions are built with properties that speakers commonly attribute to the bearers. For names like "Aristotle," "Columbus," "Einstein," the properties generally regard the great achievements of the individuals named by those names.

4 There is also a group of methodological objections claiming that Machery et al.'s works fail to collect data on semantic intuitions because the task questions of the tests can be understood ambiguously. Consequently, they may elicit intuitions about the speaker's reference or semantic reference or about the epistemic perspective of the experimenter or the epistemic perspective of the characters in the vignettes. See Ludwig (2007), Deutsch (2009), and Sytsma and Livengood (2011).
}

ette uses the name "Gödel" to talk about Gödel or Schmidt may be indicative - at the very best - of the participants' inclinations towards ways of theorising about reference determination, but it does not reveal any aspect of the real phenomenon in which philosophers are interested, namely reference determination itself.

Thus, although Devitt and Martí do consider as legitimate the idea that philosophical theories of reference can be experimentally tested, they argue that such theories should be tested not against referential intuitions but against the reality of what referential intuitions are about, i.e., linguistic usage. Experimental surveys must be conducted on speakers' linguistic performances, their production and understanding of sounds and signs in communication exchanges.

In the following section, we will develop Devitt and Martí's insight into a method for testing the causal theory of reference for proper names. We will discuss a problem that the idea of testing linguistic usage gives rise to. The problem stems from the ambiguity between semantic reference and speaker's reference.

\section{Testing linguistic usage: semantic reference vs. speaker's reference}

Our research question is a conditional one: if one agrees that experiments might have an evidential role in the philosophy of language and accepts Devitt and Martí's idea that experiments in theory of reference must collect data about linguistic usage and not about referential intuitions, then how can one test the causal theory of reference? As soon as one starts addressing this question, one faces a problem related to the distinction between semantic reference and speaker's reference. Let us elaborate on this point. In order to confirm the causal theory of reference one needs to derive from it some empirical consequences to be experimentally tested. If one accepts that experiments must collect data about linguistic usage, then in order to confirm experimentally the causal theory of reference, one needs to derive from it some empirical consequences concerning speakers' linguistic performances.

The idea that experiments can be designed to confirm the causal theory of reference by testing speakers' linguistic performances presupposes that one can derive predictions about speakers' linguistic performances in the experimental settings from the causal theory of reference. Very roughly, it is presupposed that the following methodological principle makes sense:

The causal theory of reference $T$ predicts that in experimental settings $C$ competent speakers will utter $U$ or will understand $U$ in a certain way (say by responding to it by uttering $U^{*}$ or by doing $X)$. Therefore, if in experimental settings $C$ speakers utter $U$ or understand $U$ in that way, the theory $T$ is confirmed. If in conditions $C$, speakers do not utter $U$ or do not understand $U$ in that way, the theory $T$ is disconfirmed. Similarly, if $T$ predicts that speakers will not utter $U$ or will not understand $U$ in a certain way but they do so, that disconfirms $T$; if speakers do not understand, that confirms $T$.

We claim that this methodological principle suffers from a problem. The problem is that the causal theory of reference-and in general all philosophical theories of reference-do not make any predictions about linguistic usage if taken separately from pragmatic assumptions. Linguistic usage consists of speakers' performances in the production and understanding of sounds and signs in communication exchanges. What speakers say, write or do in order to perform linguistic acts and in response to them depends on their conceptions and representations of the context of utterance, the topic of the conversation, background information, expectations about the hearer (speaker) and, of course, their (implicit) knowledge of pragmatic maxims, i.e., general principles governing cooperative behaviour. Even in the very simplified, 
controlled and circumscribed contexts of conversation such as those simulated by experimentalists when they carry out their tests, it is not possible to make predictions about the participants' linguistic performances from the causal theory of reference alone. In particular, one cannot derive from the causal theory of reference that in the experimental settings $C$ participants will perform certain linguistic acts, unless one makes a crucial assumption about the participants' pragmatic processes involved in the understanding and representation of the settings and the question tasks of the experiments. That crucial assumption regards the distinction between semantic reference and speaker's reference.

The causal theory of reference for proper names is a theory of their semantic reference. It is not a theory of how speakers use proper names. Of course, as Kripke (1977) taught us, there is a fundamental connection between semantic reference and use. When a speaker uses sincerely and literally a proper name, she has the general semantic intention to use the name to refer to its semantic bearer. However, the speaker has also a specific intention to make a reference to a particular object. As Kripke said, in simple cases, the two intentions coincide. But in complex cases, the two intentions might be different. The speaker may have an object in mind she wants to refer to, but that object may not be the semantic bearer of the name. If the speaker believes erroneously that her specific intention and her general semantic intention determine the same object, she will misuse the name to refer to that object, although that object is not the semantic bearer of the name. In such a case, the object referred to is the speaker's reference. In Kripke's famous example, a speaker looking at Smith in the distance and misidentifying him for Jones can say "Jones is raking leaves" and use the name "Jones" to make reference to Smith. Likewise, a hearer who knows that the man in the distance is Smith and not Jones will understand that the speaker made reference to Smith and said of Smith that he was raking leaves, despite the fact that the speaker uttered the name "Jones" and Smith is not its semantic reference.

The distinction between semantic reference and speaker's reference gives rise to a problem for experimentalists who want to confirm a theory of reference by testing linguistic usage. In particular, experiments need to keep the simple cases in which the specific intention coincides with the general semantic one apart from the complex cases in which the two intentions diverge. For the sake of brevity, let us call the simple cases in which the specific intention coincides with the general semantic one, cases in which the linguistic performances are guided by the semantic reference, and the complex cases in which the specific intention diverges from the general semantic one, cases in which the linguistic performances are guided by the speaker's reference.

To make the problem clear, consider the following case. Suppose that the causal theory of reference says that the semantic reference of the proper name " $\mathrm{N}$ " is the object 0 . If experiments show that in the experimental settings participants do not use "N" to refer to $o$ or do not understand utterances of " $N$ " as referring to $o$, this does not disconfirm the causal theory of reference. Likewise, finding out that participants use "N" to refer to $o$ or understand utterances of " $\mathrm{N}$ " as referring to $o$ does not confirm the causal theory of reference either. One gets a disconfirmation and respectively a confirmation of the causal theory of reference only if the participants' linguistic performances are guided by the semantic reference. This means that linguistic performances count as evidence for or against the causal theory of reference only if there is evidence that they have been guided by the semantic reference.

One way to cope with this problem is to bite the bullet and agree that it is not possible to derive predictions about speakers' linguistic performances directly from the causal theory of reference. However, from the causal theory of reference it is possible to derive predictions about speakers' linguistic performances in the following conditional form:

Nomic conditional: in the experimental settings $C$, if the participants' linguistic performances are guided by the semantic reference, then the participants will/won't utter $U$ or will/won't understand $U$ in a certain way.

In the next sections, we will present a series of experiments aimed at testing conditionals of this form with respect to people proper names and geographical proper names. In Section 3, one experiment on people proper names will be presented. In Section 4 we will argue that, although this experiment is defective since it does not solve completely the problem of the semantic/speaker's reference ambiguity, it provides data that are nonetheless sufficient to confirm the causal theory of reference for people proper names. In Section 5 we will present a second study that solves the problem of the semantic/speaker's reference ambiguity. This study not only replicates the results of the previous experiment with respect to people proper names, but it confirms the causal theory of reference also for geographical proper names.

\section{Experiment 1: People proper names}

The goal of Experiment 1 is to confirm the causal theory of reference testing linguistic usage with people proper names (PNs).

\subsection{Methods}

\subsubsection{Participants}

Thirty-one students from the University of Genoa (Italy) ( $[\mathrm{M}=24.7 ; \mathrm{SD}=3.8 ; 17$ males, 13 females) participated as volunteers or for course credits. Participants were all native Italian speakers. Only one participant was excluded from the sample because of an interruption in the task performance. None of them was aware of the goal of the experiment. Informed consent was obtained from every participant. Participants were randomly assigned to two groups: A and B.

\subsubsection{Stimuli}

We created 15 written scenarios ( 1 practice trial +6 target scenarios +8 fillers). The scenarios presented fictional ordinary circumstances. Each scenario consisted of a story, a question and three pictures.

The stories in the target scenarios presented fictional circumstances in which a description, e.g., "the painter of The Woman on the Beach," associated with a target people proper name (PN), e.g., "Marco Salvi," by a community of speakers, e.g., "the people you work for," was not satisfied by the bearer of the name and was instead satisfied by another individual bearing another name, e.g., "Eugenio Marini." Here is an example:

"Suppose you are a detective. The people you work for ask you to track down the author of the famous painting The Woman on the Beach, the young Mr. Marco Salvi. While you are conducting your inquiries, you find out that the real painter of The Woman on the Beach is not Marco Salvi, but the old Mr. Eugenio Marini." ${ }^{5}$

The stories were followed by a question asking the respondent to assign a reference to the name. The description was made explicit and presented together with the name to make the semantic contradiction explicit and to test directly whether in reference

\footnotetext{
5 Original Italian version of the probe: "Immagina di essere un investigatore. Le persone per cui lavori ti chiedono di rintracciare l'autore del famoso quadro La donna sulla spiaggia, il giovane Marco Salvi. Mentre conduci le tue ricerche scopri che in realtà il vero autore de La donna sulla spiaggia non è Marco Salvi, ma l'ormai ottantenne Eugenio Marini”.
} 
assignment, participants relied more on the name and the causal chain involving the name or on the description associated with the name ${ }^{6}$; for example:

"If you were asked to track down the painter of The Woman on the Beach, Mr. Marco Salvi, who would you look for?"7

The question was then followed by three pictures representing three alternatives: (i) the reference satisfying the description (i.e., the descriptive option); (ii) the reference of the name (i.e., the causal option); and (iii) a covered box as Other option meaning "Either both or neither of the two"-see Fig. 1.

The pictures of all the items except for the covered box were pretested to ensure that they were proper prototypical images of the reference of the name/description as described in the stories-e.g., picture 1 in Fig. 1 obtained a high rate in a norming study with a 5-point Likert scale where this picture was presented and the participants were asked to rate the extent to which it was representative of a younger man. Moreover, the position of the pictures representing the causal and the descriptive options was randomised across items.

In regard to the covered box, it is worth noting that, although it was presented in the instructions as an "Other option" standing for "Neither of the two alternatives or both of them," the only meaningful interpretation of this option in the target items was "Both of them" since the two pictures available represented the only two possible references for the name/description pair.

The 8 filler items had a structure similar to the target items with the only difference being that they were designed in such a way that: (i) two of them forced the alternative on the left (i.e., picture 1); (ii) two forced the one on the right (i.e., picture 3); (iii) two forced the other option because pictures 1 and 3 presented equally plausible alternatives; and (iv) two forced the other option because pictures 1 and 3 presented equally implausible alternatives. In this way, the 8 filler items worked as both distractor and control items. $^{8}$

As an independent variable, we manipulated the relevance of the description associated with the target name with respect to the fictional context of the story by including a sentence intended to increase the relevance of the description. For example, the relevance of the description "the painter of The Woman on the Beach" was increased/manipulated by including the following sentence (marked in italics) in the question:

If you were asked to track down the painter of "The Woman on the Beach," Mr. Marco Salvi, in order to ask him to produce a similar painting, who would you look for? ${ }^{9}$

All the critical sentences embedded in the questions to increase the relevance of the description were pretested with a paper-andpencil questionnaire administered to 16 participants. In the questionnaire, participants were presented a question asking about the likelihood of the relation between the critical sentence and the description associated with the name. Participants rated the

\footnotetext{
${ }^{6}$ The position of name/description was randomised across the items in order to avoid any possible effect due to their syntactic position, for example: (i) the postposition of the description with respect to the name that could lead to interpreting the name as the focus of the sentence forcing a causal interpretation or (ii) any other potential effect of the appositive construction.

${ }^{7}$ Original Italian version of the question in the Standard condition: "Se ti venisse chiesto di rintracciare l'autore di La donna sulla spiaggia Marco Salvi, chi cercheresti tra i due?"

${ }^{8}$ All the participants reached a $50 \%+1$ correctness level for the filler questions contained in the filler items. Consequently, no participant was excluded from the sample for having violated this accuracy criterion.

9 Original Italian version of the question in the Relevance condition: "Se ti venisse chiesto di rintracciare l'autore de La donna sulla spiaggia Marco Salvi, per far dipingere un quadro simile a La donna sulla spiaggia, chi cercheresti tra i due?"
}

probability of the event described by the critical sentence $p$ from 1 , very unlikely, to 5 , very likely. Sentences that obtained 5 were selected as critical sentences.

By manipulating the relevance of the description with the critical sentence, we created a between-subject design where two groups of participants were randomly assigned to either a Standard condition or a Relevance condition. As argued above, it is not possible to test linguistic usage by excluding the role played by pragmatic factors. In reference assignment, a crucial role is typically played by the degree of relevance of the potential referents (Clark, 2013; Wilson, 1992). We introduced the relevance condition as a control condition to verify the influence of pragmatic variables in reference assignment. Our expectation was that the high degree of relevance of the description associated with the name would prompt participants to select the descriptive reference.

\subsubsection{Procedure}

The task consisted of reading the stories and answering the questions by selecting the picture that best represented the reference for the target name. The order of the trials was randomised. The experiment was run as a paper-pencil questionnaire. Participants were tested individually at the Laboratory of Psychology of Language at the University of Genoa (Italy) in a quiet room with normal lighting.

The experiment started with written instructions informing participants that they were expected to read stories containing a name and select between two pictures the one corresponding to what they considered to be the reference of the name (or the Other option if they considered both alternatives plausible or neither of them). An example was then given, followed by a practice trial. Participants were instructed to read the stories only once. Participants spent around $18^{\prime}$ to complete the task. During the debriefing phase, no participant was found to have been aware of the goal of the experiment or to have identified the target items among the list of 14 stories.

As the experiment was run in paper-and-pencil, participants' responses were manually decoded by a first coder and then checked by a second coder and corrected if necessary.

\subsubsection{Dependent variable}

We were interested in investigating: (i) any eventual difference in percentages of choices per condition and (ii) any eventual difference in percentages of choices across conditions. Our main dependent variable was therefore the percentages of participants' choices in the target items within and across the two experimental settings.

\subsection{Results}

\subsubsection{Results within condition}

For both the Standard condition and Relevance condition, participants could provide their answer by choosing between a causal option, a descriptive option, or neither of them by selecting the other option possibility. Although in both conditions the differences in percentages of the possible options were clear-cut, we ran a chisquare analysis separately per condition to statistically compare the difference between the available options. Moreover, in order to avoid a Type I error, we applied Yates's continuity correction to the Pearson's chi-squared test. In what follows, the test statistics with Yates' correction are reported.

In the Standard condition, our participants chose the causal option $57.77 \%$ of the time and the descriptive one $26.66 \%$ of the time, while they selected the other option possibility only $15.55 \%$ of the time. The Chi-square statistics confirmed that there was a significant difference between (i) the causal and the descriptive options $\left(\chi^{2}(1)=41.61 ; \mathrm{p}<0.001\right)$; (ii) the causal and the other 

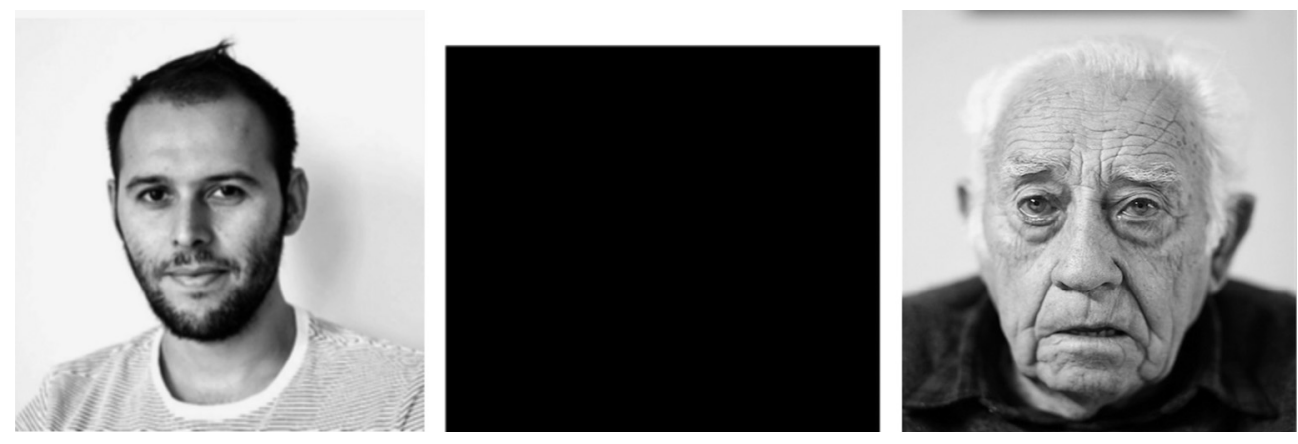

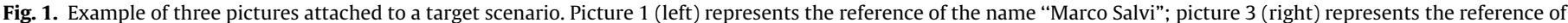
the description "The painter of The Woman on the Beach"; picture 2, the covered box (centre), stands for the "Other option."

option $\left(\chi^{2}(1)=19.96 ; \mathrm{p}<0.001\right)$; and (iii) the descriptive and the other option $\left(\chi^{2}(1)=4.52 ; \mathrm{p}<0.05\right)$.

In the Relevance condition, participants selected the causal interpretation $16.66 \%$ of the time, and the descriptive one $66.66 \%$ of the time, while they chose the other option $16.66 \%$ of the time. The Chi-square statistics confirmed that, while the difference between the causal interpretation and the other option was not significant $\left(\chi^{2}(1)=1.84 ; \mathrm{p}=\mathrm{n} . \mathrm{s}\right.$.), the percentage of choice for the descriptive option was significantly higher than that of the causal option $\left(\chi^{2}\right.$ $(1)=27.7 ; \mathrm{p}<0.001)$ and that of the other option preference $\left(\chi^{2}\right.$ (1) $=27.7 ; \mathrm{p}<0.001)$.

Overall, the results within condition suggest a reversed pattern of preference: when presented within a Standard context, people proper names were significantly given a causal interpretation but when they were supported by a Relevance context, participants substantially preferred a descriptive interpretation.

\subsubsection{Results across conditions}

The above trend of results was further confirmed in the acrossconditions comparison. Statistical analysis was performed with Linear Mixed Models (LMM) using the lme4 package (Bates et al., 2015) and the lmerTest package to extend the lmer model and provide the degrees of freedom and the p-value. The random structure of the model was constructed by using subjects and items as random factors (Baayen, Davidson, \& Bates, 2008), while participants' responses in the two conditions (i.e., Standard vs. Relevance) constituted the fixed structure of the model. The results revealed a significant effect of condition $(t=2.81, \mathrm{df}=1, \mathrm{p}<0.01)$, further confirming the reversed pattern of choices between the causal and descriptive option in the two conditions (see Fig. 2). In fact, while people proper names were significantly given a causal interpretation in the Standard condition $57.77 \%$ of the time vs. $16.66 \%$ in the Relevance condition), they were interpreted descriptively when presented within a Relevance context $(66.66 \%$ of the time vs. $26.66 \%$ in the Standard condition), which suggests that their interpretation was influenced by the degree of relevance of the description.

\section{Partial confirmation of the causal theory of reference}

In this section, we will argue that our experimental data provide a confirmation of the causal theory of reference for people proper names.

In light of what we said in Section 2, what can be predicted from the causal theory of reference is the nomic conditional that, in our experimental settings, if participants understand names as referring to their semantic reference, then they choose the causal options. Therefore, finding out that participants chose the causal option does not count as a confirmation of the theory. Those find-
EXPERIMENT 1 - PEOPLE NAMES

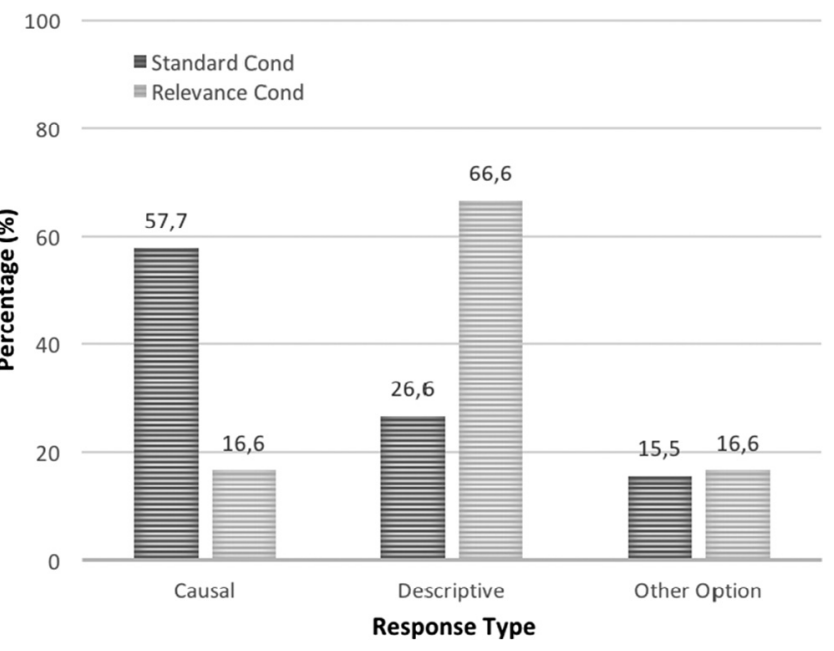

Fig. 2. Comparison of the percentage of choices in the Standard and Relevance condition with PNs

ings do not confirm the causal theory of reference unless there are reasons to believe that participants interpreted the names as referring to their semantic reference. Likewise, finding out that participants chose the descriptive option does not count as a disconfirmation of the causal theory of reference. Such findings count as a disconfirmation of the causal theory of reference only if there are reasons to rule out the possibility that participants interpreted the names as referring to the speaker's reference.

In interpreting our experimental data, then, we need to take into account both the possibility that semantic reference guided participants' choices and the possibility that the speaker's reference guided them.

First, the results confirm the causal theory of reference for people proper names because the participants' primary choice in the standard condition was the causal option, i.e., the bearer of the name. The point is that we have reasons to believe that it was the semantic reference that guided the participants' choices. In fact, from the point of view of the causal theory of reference, the only candidate consistent with the semantic reference was the causal option, while the descriptive option was the only candidate for the speaker's reference. Since we have evidence that the semantic reference guided the participants' choices, we have evidence that the antecedent of the nomic conditional is true. Our experimental data provide evidence that the consequent is true as well. Therefore, the prediction that if the semantic reference guides reference assignment, then the participants choose the bearer of the name (causal option) and not the satisfier of the description (descriptive option), is verified. The prediction of 
the causal theory of reference, in the form of the nomic conditional, is confirmed. This confirms the causal theory of reference with regard to people proper names. An important issue, however, remains open: although the majority of the participants opted for the causal option, it might be the case that the participants who opted for the descriptive option were guided by the speaker's reference. In such a case, the causal theory would be even more strongly confirmed.

In the next section, we will present a second study that resolves the problem of the semantic/speaker's reference ambiguity. It allows for separating the descriptive responses guided by the speaker's reference from those guided by the semantic reference as predicted by the classical descriptivist theory. Finding out that the choice of the descriptive option was guided by the speaker's reference will give the causal theory of reference a stronger confirmation. Indeed, the conditional prediction will be confirmed in its counterposed form: if participants choose the descriptive options, then they are not guided by the semantic reference.

\section{Experiment 2: Resolving the semantic/speaker's reference ambiguity}

\subsection{Methods}

\subsubsection{Participants}

61. Italian students (University of Genoa - Italy) participated as volunteers $[M=29.4 ;$ SD $=7.6 ; 23$ males, 38 females]. Participants were all native Italian speakers. Informed consent was obtained from every participant.

\subsubsection{Stimuli and procedure}

Similar to Experiment 1, we created 16 written scenarios. The target scenarios had a similar structure as those used in the previous experiment. The filler/control scenarios were structured as in Experiment 1 . One difference with respect to Experiment 1 was that we included in the same experiment 5 target stories testing geographical proper names (GNs) and 5 target stories testing people proper names.

Although, as we will explain in Section 5.2, the methodology used in this experiment allows us to test the causal theory of reference by investigating linguistic usage and in spite of the potential interference of pragmatic variables, because it allows us to distinguish between the participants' responses that were guided by the semantic reference from those that were guided by the speaker's reference, we have nonetheless attempted to reduce the interference of pragmatic variables. Since the Relevance condition in Experiment 1 revealed that when the pragmatic variable of the relevance of the description associated with a name was increased, participants were prompted to select the descriptive option, in our second experiment we pretested all the descriptions associated with both people and geographical proper names through a norming study. Descriptions were pretested with an online questionnaire administered to 21 participants. In the questionnaire, participants were asked to rate the likelihood of the event that someone could visit a place (testing GNs) or look for a person (testing PNs), given the content of the description associated with the name. For example, to test the relevance of the description "the birthplace of the musician Francois Morrie" associated with the geographical name "Calè," we asked, "How likely is it that someone visits a certain city for the only fact that it is a musician's birthplace?" Participants rated the probability of the described event on a 5-point Likert scale, from 1, very unlikely, to 5, very likely. The purpose of the norming study was twofold: first, to balance the level of the relevance of the descriptions; second, to make sure our stimuli received a low degree of relevance. The descriptions that obtained, on average, a score between 1 and 2 were therefore selected for the experiment.
Furthermore, with respect to Experiment 1, the structure of the stories was reduced in order to avoid the potential interference of other pragmatic variables. For example, one of the stories used with GNs was:

"In Sweden the city of Yalmo is known as the place of the 99days battle. You find out that, actually, the 99-days battle was not fought in Yalmo but in the small close island of Grund" 10

As in the previous experiments, stories were followed by a question asking the respondent to assign a reference to the name, for example:

\section{QUESTION 1:}

"If you were asked to go to Yalmo, the place of the 99-days battle, where would you go?"11

The question was then followed by two pre-tested pictures: (i) a picture representing the satisfier of the description (i.e., the descriptive option) and (ii) a picture representing the causal reference of the name (i.e., the causal option) (see Fig. 3).

Different from Experiment 1, in this experiment the first question was then followed by a second one, prompting a production task asking participants to name the chosen place. For example:

\section{QUESTION 2:}

"Suppose you visited the place you chose. You would say you have been to:"

In answering question 2, the participants were given two options:

\section{(i) Yalmo \\ (ii) Grund}

"Yalmo" is name ${ }^{1}$ whose bearer does not satisfy the description, while "Grund" is name ${ }^{2}$ whose bearer satisfies the description.

\subsubsection{Procedure}

The experiment was run online. The procedure consisted of two tasks:

Task 1-Selection Task: selecting the picture, out of two, that best represents the reference of the target name.

Task 2-Elicited Production Task: selecting the name, between name $^{1}$ and name $e^{2}$, that best reports the choice in Task 1 .

\subsection{Rationale of the study}

Experiment 2 had two tasks. In Task 1, there were two options: the causal option and the descriptive option. In Task 2, participants were asked to select a name to report their choice in Task 1 and they were given two alternatives: name ${ }^{1}$ and name ${ }^{2}$. The dependent variable of this study was the frequency of response pairs in Task 1 and Task 2 . In other words, the experiment investigated the frequency of the four possible combinations of responses in the two tasks. Let us elaborate on the rationale of our methodology. Responses in the two tasks resulted in four possible combinations: causal option/name ${ }^{1}$, causal option/name ${ }^{2}$, descriptive option/name ${ }^{1}$ and descriptive option/name ${ }^{2}$.

Causal option/name ${ }^{1}$ : if the participants selected the causal option in Task 1, e.g., the city of Yalmo, and they reported their choice using name ${ }^{1}$ in Task 2 , the name "Yalmo," then we have evidence for the causal theory of reference. The selection of the causal

\footnotetext{
10 Original Italian version of the probe: "In Svezia la città di Yalmo è nota come la località della battaglia dei 99 giorni. Scopri che in realtà la battaglia dei 99 giorni non è stata combattuta a Yalmo bensì nella piccola vicina isola di Grund

11 Original Italian version of the question: "Se ti chiedessero di recarti a Yalmo, la località della battaglia dei 99 giorni, dove andresti?"
} 

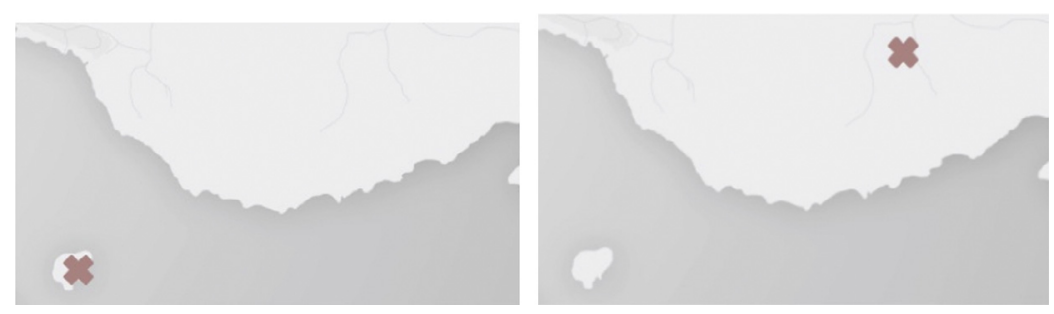

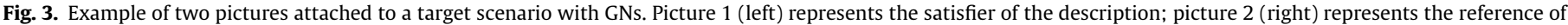
the name "Yalmo."

option in Task 1 reveals that the participants assigned to the name "Yalmo" the reference predicted by the causal theory. In reporting their choice using the name "Yalmo" in Task 2, moreover, they give evidence that they take the city of Yalmo to be the semantic reference of the name "Yalmo." This confirms the nomic conditional predicted by the causal theory of reference.

Causal option/name ${ }^{2}$ : if the participants selected the causal option in Task 1, e.g., the city of Yalmo, and they reported their choice with name ${ }^{2}$ in Task 2, the name "Grund," then we have evidence that they did not understand the scenario, in which the name "Grund" referred to the island of Grund and not to the city of Yalmo. This combination of responses represents therefore a control option, used to assess participants' comprehension of the task and their accuracy in the performance.

Descriptive option/name ${ }^{1}$ : if participants selected the descriptive option in Task 1, e.g., the island of Grund, and they opted for name ${ }^{1}$, the name "Yalmo," then we have evidence against the causal theory of reference. In this case, participants took the name "Yalmo" as referring to the island of Grund in Task 1, which is the satisfier of the description associated with the name "Yalmo." Afterwards, participants used the name "Yalmo" to refer to the island of Grund in Task 2. In doing so, they showed that they took the island of Grund to be the semantic reference of the name "Yalmo." This makes the nomic conditional false. The participants' linguistic performances were guided by the semantic reference, and they chose the descriptive option.

Descriptive option/name ${ }^{2}$ : if participants selected the descriptive option in Task 1, e.g., the island of Grund, and reported their choice using name ${ }^{2}$ in Task 2, the name "Grund," then we have evidence that confirms the causal theory of reference. Although participants chose the descriptive option, they show that their choice was guided by the speaker's reference. They understood the use of the name "Yalmo" in Task 1 as referring to the speaker's reference. The conditional prediction of the causal theory of reference is confirmed in its counterposed form: if participants chose the descriptive option, then their choice is not guided by the semantic reference. $^{12}$

\footnotetext{
12 Someone could argue that a third legitimate way to report the participants' choice in Task 1 would be to use the description itself, e.g., "I've visited the place where the 99-days battle took place." We decided, however, to exclude this third option from Task 2 because it is not directly informative about whether participants are using the name "Yalmo" consistently with the causal theory. For example: in Task 1, participants select the picture consistent with the causal option, e.g., they opt for picture 2 on the right in Fig. 3 representing the city of Yalmo as referent of the proper name "Yalmo," and in Task 2 they describe the option selected in Task 1 using the description "the place where the 99-days battle took place," the combination of responses is totally inconsistent. The description, in fact, does not fit at all with the causal option in Task 1. Consider moreover the case in which participants in Task 1 select the picture consistent with the descriptive option, e.g., picture 1 on the left in Fig. 3 representing the island, and in Task 2, they report their choice using the description "the place where the 99-days battle took place." In this case, this combination of responses would be totally uninformative: the fact they are using the description "the place where the 99-days battle took place" to refer to the island does not allow one to determine if in Task 1 they have been guided by the semantic reference or by the speaker's reference.
}

\subsection{Results}

In Experiment 2, we investigated any eventual difference in combination responses (i) between PNs and GNs and (ii) for PNs and GNs separately.

\subsubsection{Results}

Linear Mixed Models comparing the frequency of response combinations between PNs and GNs (random factors: subject, items; fixed factor: semantic domain) revealed that the two semantic domains significantly differed $(t=-2.73 \quad d f=1$; $\mathrm{p}<0.006)$-see Fig. 4 for mean frequencies in percentage.

For PNs, the chi-square statistics with Yates' continuity correction revealed that the response combination causal option/name ${ }^{1}$ significantly differed from causal option $/$ name $^{2}\left(\chi^{2}(1)=210.25\right.$; $\mathrm{p}<0.0001)$, from descriptive option/name ${ }^{1} \quad\left(\chi^{2} \quad(1)=158.42\right.$; $\mathrm{p}<0.0001)$ and from Descriptive option/name ${ }^{2}\left(\chi^{2} \quad(1)=41.64\right.$; $\mathrm{p}<0.0001)$. Causal option $/$ name $^{2}$ significantly differed from both descriptive option/name ${ }^{1}\left(\chi^{2}(1)=8.53 ; \mathrm{p}<0.05\right)$ and descriptive option/name ${ }^{2}\left(\chi^{2}(1)=82.47 ; \mathrm{p}<0.0001\right)$. Finally, descriptive option/name ${ }^{1}$ significantly differed from descriptive option/name ${ }^{2}$ $\left(\chi^{2}(1)=44.94 ; \mathrm{p}<0.0001\right)$. These results suggest that the primary choice was the response combination causal option/name ${ }^{1}$.

For GNs, results suggest a different pattern: descriptive option/name $^{2}$ was selected as the primary choice. Descriptive option/name ${ }^{2}$ significantly differed from causal option/ name ${ }^{1}\left(\chi^{2}(1)=17.67\right.$; $\mathrm{p}<0.0001)$, from descriptive option $/$ name $^{1} \quad\left(\chi^{2} \quad(1)=74.58\right.$; $\mathrm{p}<0.0001)$ and from causal option $/$ name $^{2} \quad\left(\chi^{2} \quad(1)=169.81\right.$; $\mathrm{p}<0.0001)$. Causal option/name ${ }^{1}$ significantly differed from both causal option $/$ name $^{2}\left(\chi^{2}(1)=90.44 ; \mathrm{p}<0.0001\right)$ and descriptive option/name ${ }^{1}\left(\chi^{2}(1)=20.64 ; \mathrm{p}<0.0001\right)$. Finally, causal option/name $^{2}$ significantly differed from descriptive option/name ${ }^{1}\left(\chi^{2}(1)\right.$ $=30.96 ; \mathrm{p}<0.0001$ ).

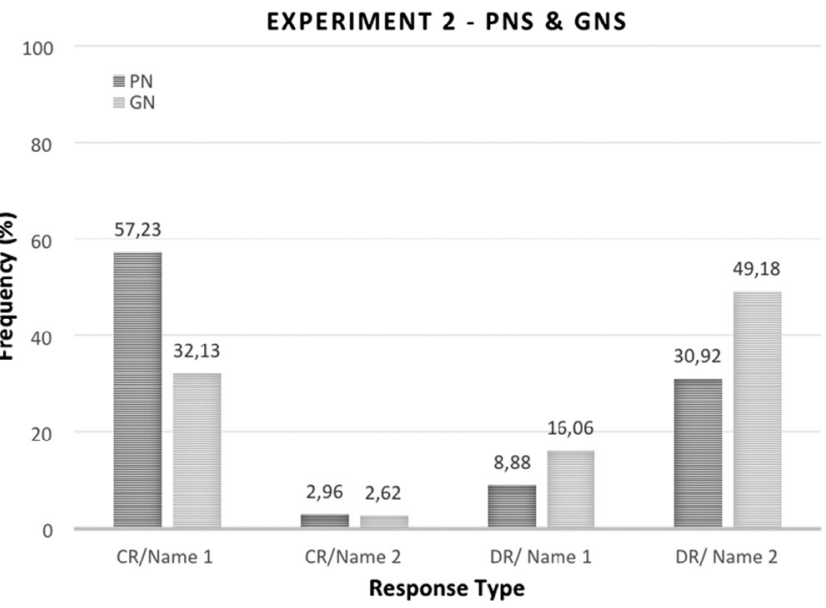

Fig. 4. Mean frequencies in percentage of response combinations $x$ response type (CR: Causal option, DR: descriptive option) and semantic domain. 


\section{Confirmation of the causal theory of reference}

We will now analyse our data and show that they confirm the causal theory of reference. Data about PNs reveal that participants opted for the combination causal option/name ${ }^{1}$ in a significant majority of the responses. This result provides evidence in favour of the causal theory of reference. When participants were asked to assign a reference to the proper name in Task 1 , they primarily assigned the semantic reference predicted by the causal theory of reference. Moreover, their responses to Task 2 show that their choice was guided by the semantic reference. The significant low percentage of selection of the combination causal option/name ${ }^{2}$ guarantees the high level of accuracy of the participants' performances. Another important result about PNs emerges clearly from Experiment 2: the combination descriptive option/name ${ }^{2}$ was selected significantly more frequently than the combination descriptive option/name ${ }^{1}$. As argued in Section 5.2, these data reveal that participants were guided by the speaker's reference in selecting the descriptive option in Task 1 . This result gives more evidence in support of the causal theory of reference and strengthens the evidence given in Experiment 1. We can conclude therefore that the causal theory of reference for PNs is confirmed.

Data about GNs show a different pattern of results. Participants performed the experiment with high accuracy, given the significant low percentage of responses causal option/name ${ }^{2}$, and they opted for the combination descriptive option/name ${ }^{1}$ significantly less frequently than the alternatives causal option/name ${ }^{1}$ and descriptive option/name ${ }^{2}$. The primary choice with GNs has been the combination descriptive option/name ${ }^{2}$, while the combination causal option/name ${ }^{1}$ has been selected less frequently. The causal theory of reference is confirmed for GNs as well. In fact, the two most frequent choices descriptive option/name ${ }^{2}$ and causal option/ name ${ }^{1}$ show that when participants chose the descriptive option they were guided by the speaker's reference and when they chose the causal option they were guided by the semantic reference, just as it is predicted by the causal theory of reference. This verifies the conditional prediction of the causal theory of reference.

If we compare the results of Experiments 1 with the results of Experiment 2, we can conclude that Experiment 2 not only replicates the data from Experiments 1, but it also confirms the hypothesis that the majority of participants that in Experiments 1 opted for the descriptive option were not guided by the descriptivist semantic reference but by the speaker's reference. This result provides a stronger confirmation of the causal theory of reference for both PNs and GNs.

\section{Differences across semantic domains}

A secondary but interesting result that emerged from Experiment 2 is that the semantic domain of a name affects reference assignment. As to PNs, participants opted for causal option/name ${ }^{1}$ as their primary choice and for descriptive option/name ${ }^{2}$ as their secondary option, while they showed the opposite trend of responses with GNs, where descriptive option/name ${ }^{2}$ was the first choice and causal option/name ${ }^{1}$ the second choice. Although our results revealed no semantic difference between PNs and GNsthe causal theory was confirmed for both-it did reveal a pragmatic difference. Where the hearer knows that the description a speaker associates with a name does not fit its semantic referent, the hearer is significantly more likely with a GN than with a PN to take the speaker to be referring to the object fitting the description rather than to the semantic referent. This pragmatic effect of the semantic domain of a name is surprising.

From a very strict psycholinguistic point of view, we can only register this difference across semantic domains. The reason why reference assignment tends to be guided by the semantic reference with PNs and by the speaker's reference with GNs may be due to anthropological, sociological and ontological aspects. Such an explanation might have to do with the kinds of objects we speak of when using PNs and GNs: speakers use names to refer to different kinds of things, to which they attribute different kinds of interests, expectations and values. Further works, however, should be designed to better investigate this second result.

\section{Conclusions}

In this paper, we have presented a method for assessing experimentally the causal theory of reference by testing linguistic usage. Although Experiment 1 revealed an interesting pattern of results, the collected data confirmed only partially the causal theory of reference due to the semantic/speaker's reference ambiguity. In Experiment 2, we proposed a method to resolve this ambiguity. The data collected in Experiment 2 confirmed the causal theory of reference for people proper names and geographical proper names.

\section{Acknowledgments}

We wish to thank all the students and $\mathrm{PhD}$ students that participated in the realization of the experiments. In particular, we thank: Davide Serpico, Eleonora Colombo, Matteo Ferrari, Giovanni Gonella, Stefano Ierardi, Davide Coraci, Matteo Baggio, Giulio Sciacca. We are indebted for constructive comments all the participants in the Experimental Semantics Workshop, Institute of Philosophy, University of Warsaw, 22-23 April 2016. In particular, we thank Michael Devitt, Nicolas Porot, Jennifer Nado, Michael Johnson, Daniel Cohnitz and Ángel Pinillos. The present work has been supported by the project EXPRESS - SIR Scientific Independence of Young Researchers financed by the Italian Ministry of Education, Universities and Research. We also thank three anonymous reviewers for their comments.

\section{Appendix A. Supplementary material}

Supplementary data associated with this article can be found, in the online version, at http://dx.doi.org/10.1016/j.cognition.2016. 12.014 .

\section{References}

Baayen, R. H., Davidson, D. J., \& Bates, D. M. (2008). Mixed-effects modelling with crossed random effects for subjects and items. Journal of Memory and Language, 59(4), 390-412.

Bates, D., Maechler, M., Bolker, B., Walker, S., Christensen, R. H. B., Singmann, H., ... Rcpp, L. (2015). Package 'lme4'. Convergence, 12, 1.

Clark, B. (2013). Relevance theory. Cambridge: Cambridge University Press.

Deutsch, M. (2009). Experimental philosophy and the theory of reference. Mind and Language, 24, 445-466.

Deutsch, M. (2010). Intuitions, counter-examples, and experimental philosophy. Review of Philosophy and Psychology, 1, 447-460.

Deutsch, M. (2015). Kripke's Gödel case. In Jussi. Haukioja (Ed.), Advances in experimental philosophy of language (pp. 7-30). London: Bloomsbury Academic.

Devitt, M. (2011a). Experimental semantics. Philosophy and Phenomenological Research, LXXXII, 418-435.

Devitt, M. (2011b). Whither experimental semantics? Theoria, 27, 5-36.

Devitt, M. (2015). Testing theories of reference. In Jussi. Haukioja (Ed.), Advances in experimental philosophy of language (pp. 31-63). London: Bloomsbury Academic.

Genone, J., \& Lombrozo, T. (2012). Concept possession, experimental semantics, and hybrid theories of reference. Philosophical Psychology, 25, 717-742.

Ichikawa, J., Maitra, I., \& Weatherson, B. (2011). In defense of a Kripkean dogma. Philosophy and Phenomenological Research, 82, 418-435.

Kripke, S. (1977). Speaker's reference and semantic reference. In P. A. French et al. (Eds.). Midwest studies in philosophy (Vol. 2, pp. 255-276). .

Kripke, S. A. (1980). Naming and necessity. Cambridge, MA: Harvard University Press.

Ludwig, K. (2007). The epistemology of thought experiments: First-person approach vs. third-person approach. Midwest Studies in Philosophy, 31, 128-159. 
Machery, E., Mallon, R., Nichols, S., \& Stich, S. P. (2004). Semantics, cross-cultural style. Cognition, 92, B1-B12.

Machery, E., Olivola, C. Y., \& de Blanc, M. (2009). Linguistic and metalinguistic intuitions in the philosophy of language. Analysis, 69, 689-694.

Mallon, R., Machery, E., Nichols, S., \& Stich, S. P. (2009). Against arguments from reference. Philosophy and Phenomenological Review, 10, 332-356.

Martí, G. (2009). Against semantic multi-culturalism. Analysis, 69, 42-48.
Martí, G. (2014). Empirical data and the theory of reference. In Topics in contemporary philosophy. In M. O’Rourke (Ed.). Reference and referring (Vol. 10). Cambridge, MA: MIT Press.

Sytsma, J., \& Livengood, J. (2011). A new perspective concerning experiments on semantic intuitions. Australasian Journal of Philosophy, 89, 315-332.

Wilson, D. (1992). Reference and relevance. UCL Working Papers in Linguistics, 4, 165-191. 